\title{
Eta Prime Gluonic Contribution to the Nucleon Self-Energy in an Effective Theory
}

\author{
A. Upadhyay ${ }^{1}$ and J. P. Singh ${ }^{2}$ \\ ${ }^{1}$ SPMS, Thapar University, Patiala, Punjab 147004, India \\ ${ }^{2}$ M.S. University of Baroda, Vadodara, Gujarat 390002, India \\ Correspondence should be addressed to A. Upadhyay; alka.iisc@gmail.com
}

Received 8 May 2014; Accepted 18 June 2014; Published 7 July 2014

Academic Editor: Alexey A. Petrov

Copyright (c) 2014 A. Upadhyay and J. P. Singh. This is an open access article distributed under the Creative Commons Attribution License, which permits unrestricted use, distribution, and reproduction in any medium, provided the original work is properly cited. The publication of this article was funded by SCOAP ${ }^{3}$.

\begin{abstract}
We estimate a possible $\eta^{\prime}$ gluonic contribution to the self-energy of a nucleon in an effective theory. The couplings of the topological charge density to nucleons give rise to OZI violating $\eta^{\prime}$-nucleon interactions. The one-loop self-energy of nucleon arising due to these interactions is studied using a heavy baryon chiral perturbation theory. The divergences have been removed using appropriate form factors. The nontrivial structure of the QCD vacuum has also been taken into account. The numerical results are sensitive to the choice of the regulator to a nonnegligible extent. We get the total contribution to the nucleon mass coming from its interaction with the topological charge density $\delta m_{\text {tot }} \cong-(2.5-7.5) \%$ of the nucleon mass.
\end{abstract}

\section{Introduction}

The axial anomaly is known to be one of the most subtle effects of the quantum field theory. The axial $U(1)$ symmetry of the QCD Lagrangian is broken by the anomaly. The corresponding pseudoscalar singlet would otherwise have a mass comparable to the pion mass. Such a particle is missing in the spectrum and the lightest candidate would be the $\eta^{\prime}$ with a mass of $958 \mathrm{MeV}$, which is considerably heavier than the octet states. Additionally, an important consequence of the axial anomaly is the fact that the would-be Goldstone boson, $\eta^{\prime}$, is massive even in the chiral limit [1]. The extra mass is induced by nonperturbative gluon dynamics [2-4] and the axial anomaly. In conventional chiral perturbation theory the $\eta^{\prime}$ is not included explicitly, although it does show up in the form of a contribution to a coupling coefficient of the Lagrangian, a so-called low-energy constant (LEC). Detailed understanding of the proton structure becomes difficult due to the complicated structure of the low-energy QCD.

The role of gluonic degrees of freedom and OZI violation in the $\eta^{\prime}$-nucleon system has been investigated through, among others, the flavor-singlet Goldberger-Treiman relation $[5,6]$, which, in the chiral limit, reads as

$$
M g_{A}^{(0)}=\sqrt{\frac{3}{2}} F_{0}\left(g_{\eta^{\prime} N N}-g_{\mathrm{QNN}}\right) .
$$

Here, $g_{A}^{(0)}$ is the flavor-singlet axial-charge measured in polarized deep inelastic scattering, $g_{\eta^{\prime} N N}$ is the $\eta^{\prime}$-nucleon coupling constant, and $g_{\mathrm{QNN}}$ is the one-particle irreducible coupling of the topological charge density $Q=\left(\alpha_{s} / 4 \pi\right) G \widetilde{G}$ to the nucleon. In (1), $M$ is the nucleon mass and $F_{0}$ renormalizes $[7,8]$ the flavor-singlet decay constant. The coupling constant $g_{\mathrm{QNN}}$ is, in part, related to $[5,6]$ the amount of spin carried by polarized gluons in a polarized proton. The large mass of $\eta^{\prime}$ and the small value of $g_{A}^{(0)}=(0.2-$ $0.35)$, extracted from deep inelastic scattering $[9,10]$, point to substantial violations of the OZI rule in the flavor-singlet $J^{P}=1^{+}$channel [11]. A large positive $g_{\mathrm{QNN}} \sim 2.45$ is one possible explanation of small value of $\left.g_{A}^{(0)}\right|_{\text {DIS }}$. 
It is important to look for significant consequences which are sensitive to $g_{\mathrm{QNN}}$. OZI violation in the $\eta^{\prime}$-nucleon system is a probe to the role of the gluons in dynamical chiral symmetry breaking in low-energy QCD. It will be interesting to calculate the nucleon self-energy due to this kind of gluonic interaction. The gluonic contribution to the nucleon selfenergy will be over and above the contributions associated with meson exchange models. It is known [12] that the pion self-energy to the nucleon is negative, and it alone contributes $(10 \%-20 \%)$ of the nucleon mass. Our objective in this work is to calculate self-energy due to this kind of gluonic interaction.

In the conventional chiral perturbation theory, the masses of the ground state baryon octet can be expanded in quark mass as [13] $\left(m_{P}^{2} \sim m_{q}\right)$

$$
M_{B}=M_{0}+\sum_{q} a_{q} m_{q}+\sum_{q} b_{q} m_{q}^{3 / 2}+\sum_{q} c_{q} m_{q}^{2}+\cdots
$$

Borasoy [14] has shown that $\eta^{\prime}$ can also be included in baryon chiral perturbation theory in a systematic way. In this approach, $\eta^{\prime}$ is included as a dynamical field variable instead of integrating it out from the effective field theory. It has a justification in $1 / N_{C}$ expansion of QCD [2-4], where the axial anomaly is suppressed by powers of $1 / N_{C}$ and $\eta^{\prime}$ appears as a ninth Goldstone boson. Since there is no anomaly in the leading order of the $1 / N_{C}$ expansion, it is natural to expect that, up to leading order, the singlet NG boson is present and degenerate with other $\left(N_{f}^{2}-1\right)$ nonsinglet NG bosons in the chiral limit. The theory at this level is thus chiral $U\left(N_{f}\right) \times$ $U\left(N_{f}\right)$ invariant. It is, however, broken by the anomaly which exists in nonleading order of the $1 / N_{C}$ expansion.

\section{The Low-Energy Effective Lagrangian}

Independent of the detailed QCD dynamics, one can construct low-energy effective chiral Lagrangians which include the effect of the anomaly and axial $U(1)$ symmetry and use these Lagrangians to study low-energy processes involving the $\eta$ and $\eta^{\prime}$ with OZI violation. In the meson sector, the $U_{A}(1)$ extended low-energy effective Lagrangian can be written as [15-17]

$$
\begin{aligned}
L_{\text {meson }}= & \frac{F_{\pi}^{2}}{4} \operatorname{Tr}\left(\partial^{\mu} U \partial_{\mu} U^{+}\right)+\frac{F_{\pi}^{2}}{4} \operatorname{Tr}\left[\chi_{0}\left(U+U^{+}\right)\right] \\
& +\frac{1}{2} i Q \operatorname{Tr}\left[\log U-\log U^{+}\right]+\frac{3}{m_{\eta_{0}}^{2} F_{0}^{2}} Q^{2},
\end{aligned}
$$

where $U=\exp \left(i\left(\phi / F_{\pi}\right)+i \sqrt{2 / 3}\left(\eta_{0} / F_{0}\right)\right)$ and $\phi=\sum_{k} \phi_{k} \lambda_{k}$. Here, $\phi_{k}$ denotes the octet of would-be Goldstone bosons $\left(\pi, K, \eta_{8}\right)$ arising out of spontaneous breaking of chiral $S U(3)_{L} \otimes S U(3)_{R}$ symmetry, $\eta_{0}$ is the singlet boson, and $Q$ is the topological charge density; $\chi=\operatorname{diag}\left[m_{\pi}^{2}, m_{\pi}^{2},\left(2 m_{k}^{2}-\right.\right.$ $\left.m_{\pi}^{2}\right)$ ] is the meson mass matrix, the pion decay constant $F_{\pi}=92.4 \mathrm{MeV}$, and $F_{0}$ renormalizes the flavor-singlet decay constant. The $U_{A}(1)$ gluonic potential involving $Q$ is constructed to reproduce the axial anomaly in the divergence of the renormalized axial-vector current [18]:

$$
\partial^{\mu} J_{\mu 5}^{(0)}=\sum_{k=1}^{f} 2 i\left(m_{k} \overline{q_{k}} \gamma_{5} q_{k}\right)+N_{f}\left[\frac{\alpha_{s}}{4 \pi} G^{\mu \nu} \widetilde{G}_{\mu \nu}\right] .
$$

and to generate the gluonic contribution to the $\eta$ and $\eta^{\prime}$ masses.

Here, $J_{\mu 5}^{(0)}=\bar{u} \gamma_{\mu} \gamma_{5} u+\bar{d} \gamma_{\mu} \gamma_{5} d+\bar{s} \gamma_{\mu} \gamma_{5} s, N_{f}=3, G_{\mu \nu}$ is the gluon field strength tensor, $\widetilde{G}_{\mu \nu}=(1 / 2) \varepsilon^{\mu \nu \alpha \beta} G_{\alpha \beta}$, and $Q(z)=$ $\left(\alpha_{s} / 4 \pi\right) G^{\mu \nu}(z) \widetilde{G}_{\mu \nu}(z)$.

The low-energy effective Lagrangian $L_{\text {meson }}$ is readily extended to include $\eta$-nucleon and $\eta^{\prime}$-nucleon couplings. The chiral Lagrangian for the meson-baryon coupling up to $O(p)$ in the meson momentum is $[7,8]$ :

$$
\begin{aligned}
L_{\mathrm{mb}}= & \operatorname{Tr} \bar{B}\left(i \gamma_{\mu} D^{\mu}-M_{0}\right) B+F \operatorname{Tr}\left(\bar{B} \gamma_{\mu} \gamma_{5}\left[a^{\mu}, B\right]\right) \\
& +D \operatorname{Tr}\left(\bar{B} \gamma_{\mu} \gamma_{5}\left\{a^{\mu}, B\right\}\right) \\
& +\frac{i}{3} K \operatorname{Tr}\left(\bar{B} \gamma_{\mu} \gamma_{5} B\right) \operatorname{Tr}\left(U^{+} \partial^{\mu} U\right) \\
& -\frac{G_{\mathrm{QNN}}}{2 M_{0}} \partial^{\mu} Q \operatorname{Tr}\left(\bar{B} \gamma_{\mu} \gamma_{5} B\right)+\frac{C}{F_{0}^{4}} Q^{2} \operatorname{Tr}(\bar{B} B) .
\end{aligned}
$$

Here, $B$ represents the baryon octet and $M_{0}$ denotes the baryon mass in the chiral limit. $D_{\mu}=\partial_{\mu}-i v_{\mu}$ is the chiral covariant derivative, $v_{\mu}=-(i / 2)\left(\xi^{+} \partial_{\mu} \xi+\xi \partial_{\mu} \xi^{+}\right)$, and $a_{\mu}=$ $-(i / 2)\left(\xi^{+} \partial_{\mu} \xi-\xi \partial_{\mu} \xi^{+}\right)$where $\xi=U^{1 / 2}$. The $S U(3)$ couplings are $F=0.459 \pm 0.008$ and $D=0.798 \pm 0.008$. The axial-vector current has an expansion

$$
a_{u}=-\frac{1}{2 F_{\pi}} \partial_{\mu} \phi-\frac{1}{2 F_{0}} \sqrt{\frac{2}{3}} \partial_{\mu} \eta_{0}+\cdots .
$$

In continuum QCD, dynamical chiral symmetry breaking is normally studied using Dyson-Schwinger equation for quark and gluon Green's function [19]. In low-energy effective theories, given by (3) and (5), a flavor independent selfenergy of baryon will arise due to interactions of baryons with the topological charge density $Q$ which is flavor- and colorsinglet object. This gluonic term $Q$ has no kinetic energy term, but it mixes with $\eta_{0}$ to generate gluonic mass term for $\eta^{\prime}$. The determination of masses of the physical $\eta$ and $\eta^{\prime}$ mesons also requires diagonalization of the $\left(\eta_{8}, \eta_{0}\right)$ mass matrix. Thus, part of $\eta$ mass is also generated by the gluonic term $Q[20]$.

The relativistic framework including baryons poses problem due to the existence of a new mass scale, namely, the baryons mass in the chiral limit $M_{0}$; a strict chiral counting scheme, that is, a one-to-one correspondence between the meson loops and the chiral expansion, does not exist. In order to overcome this problem, one integrates out the heavy degrees of freedom of the baryons, similar to a FoldyWouthuysen transformation, so that a chiral counting scheme emerges. Observables can then be expanded simultaneously in the Goldstone boson octet masses and the $\eta^{\prime}$ mass that 
does not vanish in the chiral limit. One obtains a one-to-one correspondence between the meson loops and the expansion in their masses and derivatives both for octet and singlet [14].

After integrating out the heavy degrees of freedom of the baryons from the effective theory [21] and assigning a fourvelocity $v$ to the baryons, the heavy baryon Lagrangian to the order we are working reads as

$$
\begin{aligned}
L_{\mathrm{mb}}= & \operatorname{Tr}(\bar{B} i v \cdot D B)+2 F \operatorname{Tr}\left(\bar{B} S_{\mu}\left[a^{\mu}, B\right]\right) \\
& +2 D \operatorname{Tr}\left(\bar{B} S_{\mu}\left\{a^{\mu}, B\right\}\right)+2 \frac{i}{3} K \operatorname{Tr}\left(\bar{B} S_{\mu} B\right) \operatorname{Tr}\left(U^{+} \partial^{\mu} U\right) \\
& -\frac{G_{\mathrm{QNN}}}{M_{0}}\left(\partial^{\mu} \mathrm{Q}\right) \operatorname{Tr}\left(\bar{B} S_{\mu} B\right)+\frac{C}{F_{0}^{4}} Q^{2} \operatorname{Tr}(\bar{B} B),
\end{aligned}
$$

where $S_{\mu}=(i / 2) \gamma_{5} \sigma_{\mu \nu} v^{\nu}$ is the Pauli-Lubanski spin vector.

In this work, our objective is to calculate the self-energy of nucleon arising due to gluonic terms within the framework of heavy baryon chiral perturbation theory including $\eta^{\prime}$. Heavy baryon chiral perturbation theory is the effective field theory of the standard model at low energies in the baryonic sector which can be successfully applied with Goldstone bosons included. However, traditional SU(3) heavy baryon chiral perturbation theory does not appear to work well. The leading nonanalytic component from loop corrections destroys the good experimental agreement which exists at lower order. The additional contributions have to be compensated by higher order counter terms. This leads to the problem with convergence of chiral series, and it can be solved using some kind of cutoff regularization instead of common dimensional regularization scheme. Here, dimensionally regularized Feynman diagrams carry implicit and large contributions from short distance physics. In contrast, the cutoff scheme picks out the long distance part of the integral, which behaves, as expected, on physical grounds.

Here, we restrict ourselves to the one-loop diagrams of the $\eta$ and $\eta^{\prime}$ with the vertices arising due to gluonic interactions with the baryons. For this purpose, we use the following matrix elements $[22,23]$ :

$$
\begin{aligned}
& \langle 0|Q| \eta\rangle=\frac{1}{\sqrt{3}} m_{\eta}^{2}\left(f_{8} \cos \theta-\sqrt{2} f_{0} \sin \theta\right), \\
& \left\langle 0|Q| \eta^{\prime}\right\rangle=\frac{1}{\sqrt{3}} m_{\eta^{\prime}}^{2}\left(f_{8} \sin \theta+\sqrt{2} f_{0} \cos \theta\right),
\end{aligned}
$$

where $\theta$ represents the singlet-octet mixing angle parameterized as follows:

$$
\begin{gathered}
\left\langle 0\left|J_{\mu 5}^{(8)}\right| \eta(p)\right\rangle=2 i f_{8} \cos \theta p_{\mu}, \\
\left\langle 0\left|J_{\mu 5}^{(8)}\right| \eta^{\prime}(p)\right\rangle=2 i f_{8} \sin \theta p_{\mu}, \\
\left\langle 0\left|J_{\mu 5}^{(0)}\right| \eta(p)\right\rangle=-\sqrt{6} i f_{0} \sin \theta p_{\mu}, \\
\left\langle 0\left|J_{\mu 5}^{(0)}\right| \eta^{\prime}(p)\right\rangle=\sqrt{6} f_{0} \cos \theta p_{\mu}, \\
J_{\mu 5}^{(8)}=\frac{1}{\sqrt{3}}\left(\bar{u} \gamma_{\mu} \gamma_{5} u+\bar{d} \gamma_{\mu} \gamma_{5} d-2 \bar{s} \gamma_{\mu} \gamma_{5} s\right) .
\end{gathered}
$$

The terms contributing to the mass of the nucleon, $\delta m_{N}$, is given in terms of the mass of $\eta$ and $\eta^{\prime}$ mesons for monopole, dipole, exponential, and sharp cutoff regularization schemes and is written as follows.

For the monopole case,

$$
\begin{aligned}
\delta m_{N}=-\frac{g_{\mathrm{QNN}}^{2}}{m^{2}} \frac{\Lambda^{4}}{64 \pi} & {\left[\left(\frac{2 m_{\eta}+\Lambda}{\left(m_{\eta}+\Lambda\right)^{2}}\right) m_{\eta^{4}}^{4} M^{2}\right.} \\
& \left.+\left(\frac{2 m_{\eta^{\prime}}+\Lambda}{\left(m_{\eta^{\prime}}+\Lambda\right)^{2}}\right) m_{\eta^{\prime}}^{4} M^{\prime 2}\right] \\
-\frac{\varepsilon \Lambda^{4}}{16 \pi^{2} F_{0}^{4}}\left[\frac{m_{\eta}^{4} M^{2}}{\Lambda^{2}-m_{\eta}^{2}}\left\{1+\frac{m_{\eta}^{2}}{m_{\eta}^{2}-\Lambda^{2}} \ln \frac{\Lambda^{2}}{m_{\eta}^{2}}\right\}\right. & \\
& \left.+\frac{m_{\eta^{\prime}}^{4} M^{\prime 2}}{\Lambda^{2}-m_{\eta^{\prime}}^{2}}\left\{1+\frac{m_{\eta^{\prime}}^{2}}{m_{\eta^{\prime}}^{2}-\Lambda^{2}} \ln \frac{\Lambda^{2}}{m_{\eta^{\prime}}^{2}}\right\}\right],
\end{aligned}
$$

for the dipole case,

$$
\begin{gathered}
\delta m_{N} \\
=-\frac{g_{\mathrm{QNN}}^{2}}{m^{2}} \frac{\Lambda^{4}}{16^{2} \pi}\left[m_{\eta}^{4} M^{2}\left(\frac{\Lambda}{2\left(m_{\eta}+\Lambda\right)^{2}}+\frac{\Lambda^{2} m_{\eta}}{\left(m_{\eta}+\Lambda\right)^{4}}\right)\right. \\
\left.+m_{\eta^{\prime}}^{4} M^{\prime 2}\left(\frac{\Lambda}{2\left(m_{\eta^{\prime}}+\Lambda\right)^{2}}+\frac{\Lambda^{2} m_{\eta^{\prime}}}{\left(m_{\eta^{\prime}}+\Lambda\right)^{4}}\right)\right] \\
-\frac{\varepsilon \Lambda^{4}}{16 \pi^{2} F_{0}^{4}}\left[\frac{m_{\eta}^{4} M^{2}}{\Lambda^{2}-m_{\eta}^{2}}\left\{1+\frac{m_{\eta}^{2}}{m_{\eta}^{2}-\Lambda^{2}} \ln \frac{\Lambda^{2}}{m_{\eta}^{2}}\right\}\right. \\
\left.+\frac{m_{\eta^{\prime}}^{4} M^{\prime 2}}{\Lambda^{2}-m_{\eta^{\prime}}^{2}}\left\{1+\frac{m_{\eta^{\prime}}^{2}}{m_{\eta^{\prime}}^{2}-\Lambda^{2}} \ln \frac{\Lambda^{2}}{m_{\eta^{\prime}}^{2}}\right\}\right],
\end{gathered}
$$

for the exponential case,

$$
\begin{aligned}
& \delta m_{N}=-\frac{g_{\mathrm{QNN}}^{2}}{32 m^{2} \pi^{3 / 2}}[ m_{\eta}^{4} M^{2}\left(\frac{\Lambda^{3}}{2}-\Lambda m_{\eta}^{2}+\sqrt{\pi} m_{\eta}^{3} e^{m_{\eta}^{2} / \Lambda^{2}}\right) \\
& \times\left[1-\phi\left(\frac{m_{\eta}}{\Lambda}\right)\right]+m_{\eta^{\prime}}^{4} M^{\prime 2} \\
& \otimes\left(\frac{\Lambda^{3}}{2}-\Lambda m_{\eta^{\prime}}^{2}+\sqrt{\pi} m_{\eta^{\prime}}^{3} e^{m_{\eta^{\prime}}^{2} / \Lambda^{2}}\right) \\
&\left.\times\left[1-\phi\left(\frac{m_{\eta^{\prime}}}{\Lambda}\right)\right]\right] \\
& \frac{\varepsilon}{16 \pi^{2} F_{0}^{4}}\left[\begin{array}{l}
m_{\eta}^{4} M^{2}\left\{\Lambda^{2}+m_{\eta^{2}}^{2} e^{m_{\eta}^{2} / \Lambda^{2}} E_{i}\left(-\frac{m_{\eta}^{2}}{\Lambda^{2}}\right)\right\} \\
+
\end{array}\right. \\
& m_{\eta^{\prime}}^{4} M^{\prime 2} \\
&\left.\times\left\{\Lambda^{2}+m_{\eta^{\prime}}^{2} e^{m_{\eta^{\prime}}^{2} / \Lambda^{2}} E_{i}\left(-\frac{m_{\eta^{\prime}}^{2}}{\Lambda^{2}}\right)\right\}\right],
\end{aligned}
$$




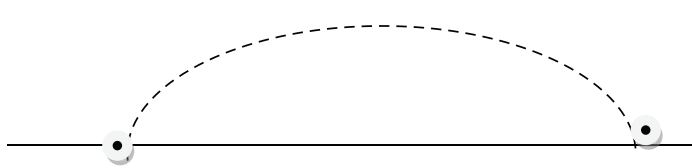

(a)

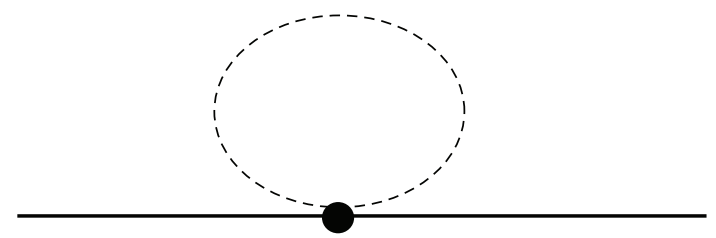

(b)

Figure 1: (a) Self-energy diagram and (b) tadpole diagram.

TABLE 1: Self-energy of a nucleon, $\delta m_{N}$, arising due to its interactions with the topological charge density in monopole, dipole, exponential, and sharp cutoff schemes as a function of regulator scale $\Lambda . \eta-\eta^{\prime}$ mixing angle $\theta$ is taken as $-18.5^{\circ}$. In dipole and exponential regularizations, in the tadpole diagram the form factor $u$ (which appears at each vertex in the self-energy diagram), $u^{3 / 2}$ or $u^{2}$, has been used. Numerical values of $\Lambda$ and $\delta m_{N}$ are in $\mathrm{GeV}$ unit.

\begin{tabular}{|c|c|c|c|c|c|c|c|c|}
\hline \multirow{3}{*}{$\Lambda(\mathrm{GeV})$} & \multicolumn{8}{|c|}{$\delta m(\mathrm{GeV})$} \\
\hline & \multirow{2}{*}{ Monopole } & \multicolumn{3}{|c|}{ Dipole } & \multicolumn{3}{|c|}{ Exponential } & \multirow{2}{*}{ Sharp cutoff } \\
\hline & & $u$ & $u^{3 / 2}$ & $u^{2}$ & u & $u^{3 / 2}$ & $u^{2}$ & \\
\hline 0.6 & -0.033 & -0.025 & -0.015 & -0.004 & -0.050 & -0.027 & -0.017 & -0.013 \\
\hline 0.8 & -0.078 & -0.055 & -0.036 & -0.010 & -0.112 & -0.062 & -0.040 & -0.036 \\
\hline 1.0 & -0.148 & -0.100 & -0.071 & -0.021 & -0.206 & -0.118 & -0.078 & -0.076 \\
\hline 1.2 & -0.248 & -0.161 & -0.121 & -0.037 & -0.334 & -0.198 & -0.135 & -0.136 \\
\hline
\end{tabular}

and for the sharp cut off case,

$$
\begin{aligned}
\delta m_{N}=- & \frac{g_{\mathrm{QNN}}^{2}}{16 m^{2} \pi^{2}}\left[m_{\eta}^{4} M^{2}\left(\frac{\Lambda^{3}}{2}-\Lambda m_{\eta}^{2}+m_{\eta^{3}}^{3} \tan ^{-1} \frac{\Lambda}{m_{\eta}}\right)\right. \\
& +m_{\eta^{\prime}}^{4} M^{\prime 2} \\
& \left.\times\left(\frac{\Lambda^{3}}{3}-\Lambda m_{\eta^{\prime}}^{2}+m_{\eta^{\prime}}^{3} \tan ^{-1} \frac{\Lambda}{m_{\eta^{\prime}}}\right)\right] \\
- & {\left[\frac{\varepsilon}{16 \pi^{2} F_{0}^{4}} m_{\eta}^{4} M^{2}\left\{\Lambda^{2}-m_{\eta}^{2} \ln \left(\frac{\Lambda^{2}+m_{\eta}^{2}}{m_{\eta}^{2}}\right)\right\}\right.} \\
& \left.+m_{\eta^{\prime}}^{4} M^{\prime 2}\left\{\Lambda^{2}-m_{\eta^{\prime}}^{2} \ln \left(\frac{\Lambda^{2}+m_{\eta^{\prime}}^{2}}{m_{\eta^{\prime}}^{2}}\right)\right\}\right]
\end{aligned}
$$

where

$$
\begin{aligned}
M^{2} & =\frac{\left(f_{8} \cos \theta-\sqrt{2} f_{0} \sin \theta\right)^{2}}{6}, \\
M^{\prime 2} & =\frac{\left(f_{8} \sin \theta-\sqrt{2} f_{0} \cos \theta\right)^{2}}{6} .
\end{aligned}
$$

\section{Regularization and the Self-Mass}

Both the one-loop diagrams given by Figures 1(a) and 1(b) are divergent. However, we must remember that we are working in an effective field theory which uses the degrees of freedom and the interactions which are correct only at low energy. It has been shown by Donoghue et al. [13] that any incorrect loop contribution coming from short distance physics can be compensated for by a shift of the parameters of the Lagrangian. Our choice of ultraviolet regulator, which represents a separation scale of long distance physics from the short distance physics, will be dictated by phenomenological considerations. In baryon chiral perturbation theory, which deals with baryons and Goldstone bosons, the separation scale is taken as $\sim 1 \mathrm{fm} \mathrm{[13]} \mathrm{corresponding} \mathrm{to} \mathrm{the} \mathrm{measured} \mathrm{size}$ of a baryon. For our problem, we consider an average "gluonic transverse size" of nucleon [24] $\left\langle\rho^{2}\right\rangle \approx 0.24 \mathrm{fm}^{2}$ corresponding to a dipole parameterization:

$$
H_{g}(x, t) \alpha\left(1-\frac{t}{m_{g}^{2}}\right)^{-2}, \quad m_{g}^{2}=1.1 \mathrm{GeV}^{2}, x \cong 10^{-1}
$$

This gives a two-gluon form factor, which we denote by $u$, of a nucleon [25] and can be used in the self-energy diagram. Another way to look at this problem is that the $U_{A}(1)$ gluonic potential involving the topological charge density leads to a contact interaction at a "short distance" $(\sim 0.2 \mathrm{fm})$ where glue is excited in the interaction region $[7,8]$ of the proton-proton collision and then evolves to become an $\eta$ or $\eta^{\prime}$ in the final state. This will lead to a sharp cutoff at an energy scale $\sim 1 \mathrm{GeV}$.

In the tadpole diagram, we will be using $u, u^{2}$, and $u^{3 / 2}$ (geometric mean of the first two), since the phenomenology does not provide any clear cut rule for this. Similarly, three types of form factors will be used in the tadpole diagram for exponential regularization also. Since the use of $u$ in the tadpole diagram does not remove the divergence while $u^{3 / 2}$ remains analytic in a restricted region, hence, we use only $u^{2}$ for the monopole case. Specifically, our form factor $u(k)$ 
TABLE 2: Self-energy of a nucleon, $\delta m_{N}$, in $\mathrm{GeV}$ unit, as a function of regulator scale $\Lambda$ for the same form factors as in Table 1 , but for $\theta=-30.5^{\circ}$.

\begin{tabular}{|c|c|c|c|c|c|c|c|c|}
\hline \multirow{3}{*}{$\Lambda(\mathrm{GeV})$} & \multicolumn{8}{|c|}{$\delta m_{N}(\mathrm{GeV})$} \\
\hline & \multirow{2}{*}{ Monopole } & & \multicolumn{3}{|c|}{ Exponential } & \multirow{2}{*}{ Sharp cutofl } \\
\hline & & $u$ & $u^{3 / 2}$ & $u^{2}$ & $u$ & $u^{3 / 2}$ & $u^{2}$ & \\
\hline 0.6 & -0.012 & -0.044 & -0.006 & -0.002 & -0.019 & -0.011 & -0.007 & -0.006 \\
\hline 0.8 & -0.027 & -0.088 & -0.015 & -0.004 & -0.042 & -0.025 & -0.017 & -0.016 \\
\hline 1.0 & -0.049 & -0.148 & -0.027 & -0.009 & -0.073 & -0.045 & -0.032 & -0.030 \\
\hline 1.2 & -0.080 & -0.225 & -0.044 & -0.014 & -0.115 & -0.073 & -0.052 & -0.051 \\
\hline
\end{tabular}

TABLE 3: Self-energy of a nucleon, $\delta m_{N}$, in dimensional regularization $(\overline{M S})$ scheme, as a function of renormalization scale $\mu$.

\begin{tabular}{lcc}
\hline & $\delta m_{N}(\mathrm{GeV})$ & \\
$\mu(\mathrm{GeV})$ & $\theta=-18.5^{\circ}$ & $\theta=-30.5^{\circ}$ \\
\hline 0.5 & -0.260 & -0.099 \\
0.7 & -0.163 & -0.062 \\
1.0 & -0.060 & -0.023 \\
\hline
\end{tabular}

for monopole, dipole, and exponential regularization has the form, respectively:

$$
\begin{aligned}
& u(k)=\frac{\Lambda^{2}}{\left(\Lambda^{2}-k^{2}\right)}, \\
& u(k)=\frac{\Lambda^{4}}{\left(\Lambda^{2}-k^{2}\right)^{2}}, \\
& u(k)=\exp \left(\frac{k^{2}}{\Lambda^{2}}\right) .
\end{aligned}
$$

Dimensional regularization scheme is not particularly suitable for effective field theories since it gets large contributions from short distance physics [13]. We have displayed our numerical results for the self-mass of the nucleon coming from both Figures $1(\mathrm{a})$ and $1(\mathrm{~b}), \delta m_{N}$, in Tables 1 and 2 . As discussed above, if we consider the regulator mass $\Lambda \cong 1 \mathrm{GeV}$ for the dipole and the sharp cut-off regularization schemes on phenomenological ground, we observe that $\delta m_{N}$ for dipole ( $u^{3 / 2}$-column), exponential ( $u^{2}$-column), and sharp cutoff schemes are approximately same for each mixing angle. Furthermore, $\delta m_{N}$ for monopole form factor is related to that for exponential form factor (both for $u^{2}$-columns) by their regulator scales [12]:

$$
\Lambda_{\text {exp }} \cong \sqrt{2} \Lambda_{\text {mon }}
$$

Hence, we take

$$
\begin{aligned}
\delta m_{N} & \cong-0.076 \mathrm{GeV}\left(\theta=-18.5^{\circ}\right), \\
& \cong-0.030 \mathrm{GeV}\left(\theta=-30.5^{\circ}\right) .
\end{aligned}
$$

Self-energy of a nucleon $\delta m_{N}$, in dimensional regularization $(\overline{M S})$ scheme, as a function of renormalization point $\mu$, has been calculated for three values of $\mu=0.5,0.7$, and $1.0 \mathrm{GeV}$ for two values of theta and shown in Table $3 . \eta-\eta^{\prime}$ mixing angle $\theta$ is taken as $-18.5^{\circ}$ and $-30.5^{\circ}$ for different regulator scales $\Lambda$. If we take the nontrivial structure of the QCD vacuum into account then, in the last term of (7), we can make the replacement $Q^{2} \rightarrow\left\langle Q^{2}\right\rangle+Q^{2} \cdot\left\langle Q^{2}\right\rangle$ can be calculated using vacuum saturation hypothesis:

$$
\left\langle\mathrm{Q}^{2}\right\rangle=\left(\frac{-1}{384}\right)\left\langle\frac{\alpha_{s}}{\pi} \mathrm{G}^{2}\right\rangle^{2} \cong-\left(\frac{1}{384}\right)(0.012)^{2} \mathrm{GeV}^{8}
$$

where, for the gluon condensate, we have used the numerical values used by ITEP group [26]. This gives a positive contribution to the nucleon mass:

$$
\delta m_{N}^{(0)} \cong+0.007 \mathrm{GeV} .
$$

Taking this into account, we get the total contribution to the nucleon mass coming from its interaction with the topological charge density $\left(\delta m_{N}\right)_{\text {tot }} \cong-(2.5-7.5) \%$ of the nucleon mass. It is known that the one-loop pion contribution to the nucleon mass is $\left(\delta m_{N}\right)_{\text {pion }} \cong-(10-20) \%$ of the nucleon mass [12]. Unlike $\left(\delta m_{N}\right)_{\text {pion }},\left(\delta m_{N}\right)_{\text {tot }}$ is flavor independent and is the same for all the members of the octet baryon family. This kind of contribution to baryon mass will not arise in the models with quark-meson interaction only. It is known that the color-magnetic-field energy in the nucleon is negative [27]. Other phenomenological applications, where the precise decoupling mechanism of the massless gluonic mode by the QCD gauge invariance and the nontrivial vacuum structure have been discussed, are in [27-32]. We have not talked about the role of scalar and tensor gluoniums in effective field theories. In particular, scalar gluonium can give rise to Higgstype mechanism, but this is beyond the scope of the present work.

\section{Conflict of Interests}

The authors declare that there is no conflict of interests regarding the publication of this paper.

\section{References}

[1] S. Weinberg, "The U(1) problem," Physical Review D, vol. 11, article 3583, 1975.

[2] G. Veneziano, “U(1) without instantons," Nuclear Physics B, vol. 159, no. 1-2, pp. 213-224, 1979. 
[3] G. Veneziano, "Goldstone mechanism from gluon dynamics," Physics Letters B, vol. 95, no. 1, pp. 90-92, 1980.

[4] E. Witten, "Current algebra theorems for the U(1) Goldstone boson,” Nuclear Physics B, vol. 156, no. 2, pp. 269-283, 1979.

[5] G. Veneziano, "Is there a QCD "spin crisis"?" Modern Physics Letters A, vol. 4, no. 17, Article ID 1605, 1989.

[6] G. M. Shore and G. Veneziano, "The U(1) Goldberger-Treiman relation and the proton "spin": a renormalisation group analysis," Nuclear Physics B, vol. 381, no. 1-2, pp. 23-65, 1992.

[7] S. D. Bass, "Gluons and the $\eta^{\prime}$-nucleon coupling constant," Physics Letters B, vol. 463, pp. 286-292, 1999.

[8] S. D. Bass, "Gluonic Effects in $\eta$ and $\eta$ 'Physics," Physica Scripta, vol. T99, article 96, 2002.

[9] S. D. Bass, "Constituent quarks and $g$ 1," The European Physical Journal A, vol. 5, no. 1, pp. 17-36, 1999.

[10] R. Windmolders, "Review of recent results in spin physics," Nuclear Physics B-Proceedings Supplements, vol. 79, pp. 51-64, 1999.

[11] G. Veneziano, CERN preprint TH-5840, 1990.

[12] M. B. Hecht, C. D. Roberts, M. Oettel, A. W. Thomas, S. M. Schmidt, and P. C. Tandy, "Nucleon mass and pion loops," Physical Review C, vol. 65, Article ID 055204, 2002.

[13] J. F. Donoghue, B. R. Holstein, and B. Borasoy, "SU(3) baryon chiral perturbation theory and long distance regularization," Physical Review D, vol. 59, Article ID 036002, 1999.

[14] B. Borasoy, "The $\eta^{\prime}$ in baryon chiral perturbation theory," Physical Review D, vol. 61, Article ID 014011, 1999.

[15] P. Di Vecchia and G. Veneziano, "Chiral dynamics in the large N limit," Nuclear Physics B, vol. 171, no. 3, pp. 253-272, 1980.

[16] C. Rosenzweig, J. Schechter, and C. G. Trahern, "Is the effective Lagrangian for quantum chromodynamics a model?” Physical Review D, vol. 21, no. 12, pp. 3388-3392, 1980.

[17] P. Nath and R. Arnowitt, “The U(1) problem: current algebra and the theta vacuum," Physical Review D, vol. 23, article 473, 1981.

[18] S. L. Adler, "Axial-vector vertex in spinor electrodynamics," Physical Review, vol. 177, no. 5, pp. 2426-2438, 1969.

[19] C. D. Roberts and A. G. Williams, "Dyson-Schwinger equations and their application to hadronic physics," Progress in Particle and Nuclear Physics, vol. 33, pp. 477-575, 1994.

[20] S. D. Bass, "Gluonic effects in eta'-nucleon interactions," In press, http://arxiv.org/abs/hep-ph/0108187.

[21] T. Mannel, W. Roberts, and Z. Ryzak, "A derivation of the heavy quark effective lagrangian from QCD," Nuclear Physics B, vol. 368, pp. 204-217, 1992.

[22] P. Ball, J.-M. Frere, and M. Tytgat, "Phenomenological evidence for the gluon content of $\eta$ and $\eta^{\prime}$," Physics Letters B, vol. 365, pp. 367-376, 1996.

[23] N. F. Nasrallah, "Erratum: Glue content and mixing angle of the $\eta-\eta \eta^{\prime}$ system: the effect of the isoscalar continuum," Physical Review D, vol. 72, Article ID 019903, 2005.

[24] M. Strikman and C. Weiss, “The nucleon's gluonic transverse size: from exclusive J/psi photoproduction to high-energy pp collisions," http://arxiv.org/abs/hep-ph/0408345.

[25] L. Frankfurt and M. Strikman, "Two gluon form-factor of the nucleon and J/psi photoproduction," Physical Review D, vol. 66, Article ID 031502, 2002.

[26] M. A. Shifman, A. I. Vainshtein, and V. I. Zakharov, "QCD and resonance physics. Applications," Nuclear Physics B, vol. 147, no. 5, pp. 448-518, 1979.
[27] X. Ji, "QCD analysis of the mass structure of the nucleon," Physical Review Letters, vol. 74, no. 7, pp. 1071-1074, 1995.

[28] K. Kawarabayashi and N. Ohta, "The $\eta$ problem in the large- $N$ limit: effective Lagrangian approach," Nuclear Physics B, vol. 175, pp. 477-492, 1980.

[29] K. Kawarabayashi and N. Ohta, "On the partical conservation of the U(1) current," Progress of Theoretical Physics, vol. 66, no. 5, pp. 1789-1802, 1981.

[30] H. Hata, T. Kugo, and N. Ohta, "Skew-symmetric tensor gauge field theory dynamically realized in the QCD U(1) channel," Nuclear Physics B, vol. 178, no. 3, pp. 527-544, 1981.

[31] N. Ohta, "Vacuum structure and chiral charge quantization in the large $N$ limit," Progress of Theoretical Physics, vol. 66, no. 4, pp. 1408-1421, 1981.

[32] N. Ohta, "Vacuum structure and chiral charge quantization in the large $N$ limit," Progress of Theoretical Physics, vol. 67, no. 3, p. $993,1982$. 

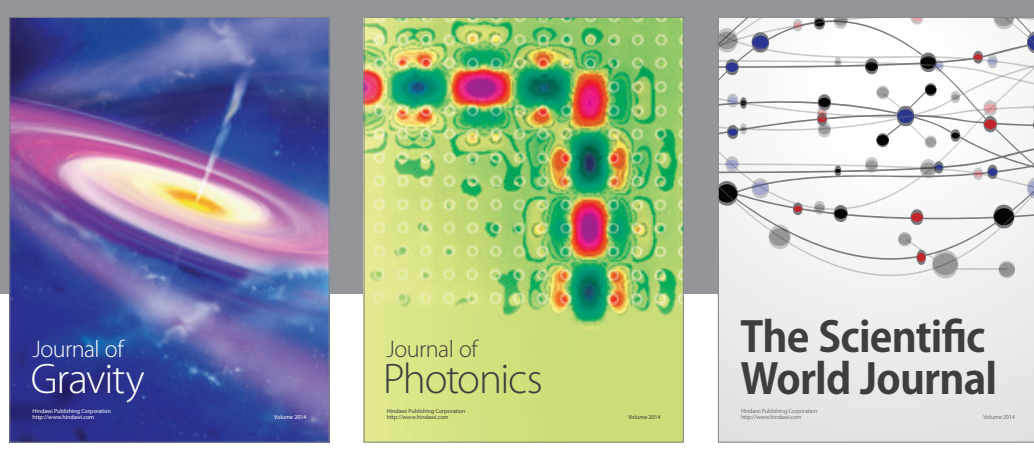

The Scientific World Journal
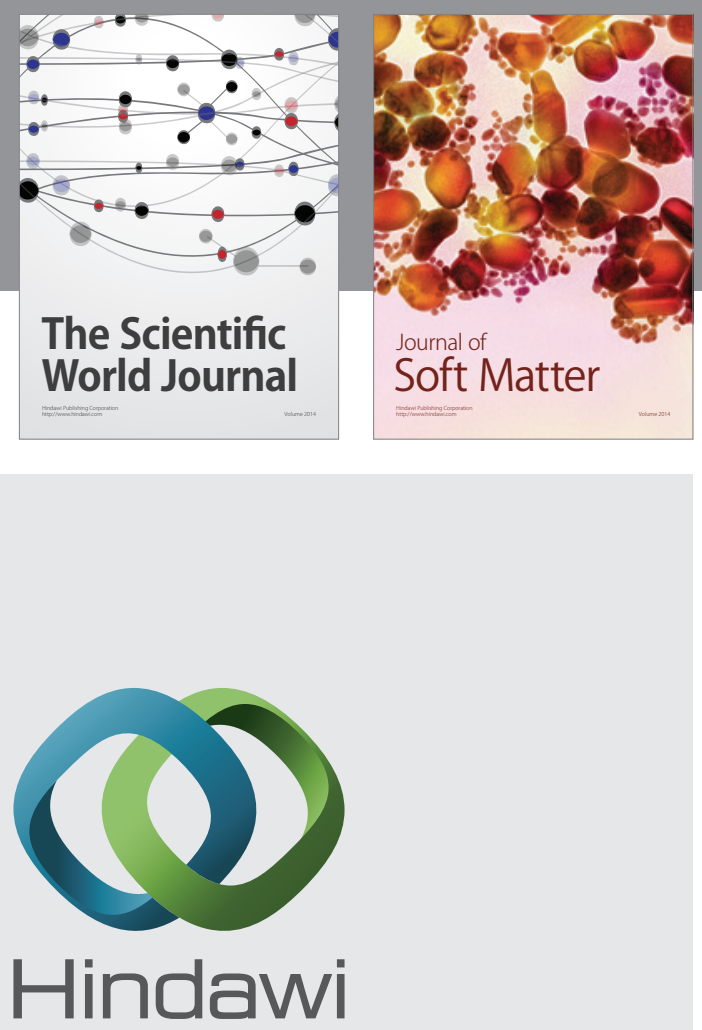

Submit your manuscripts at

http://www.hindawi.com

nternational Journal of

Statistical Mechanics
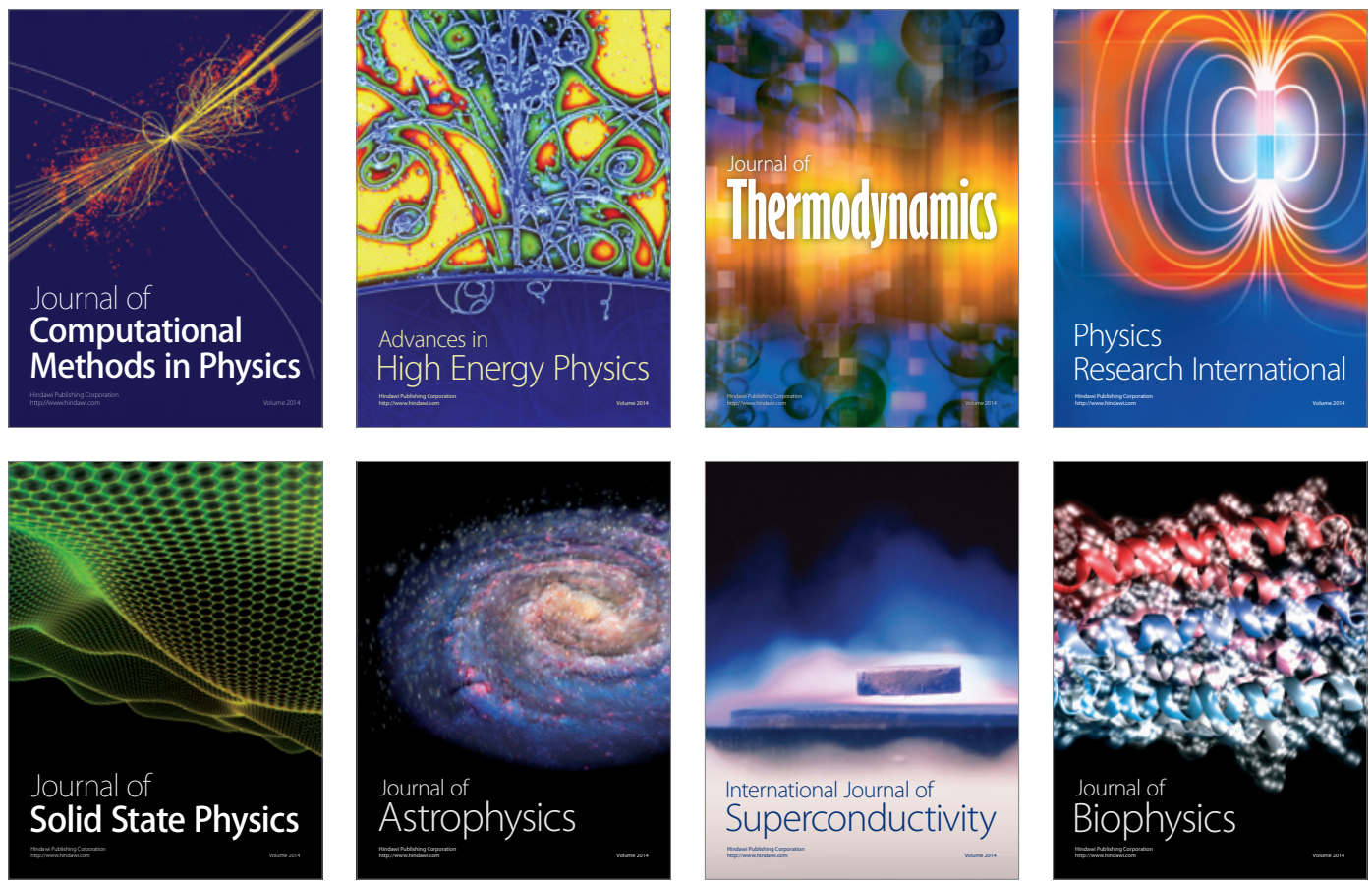
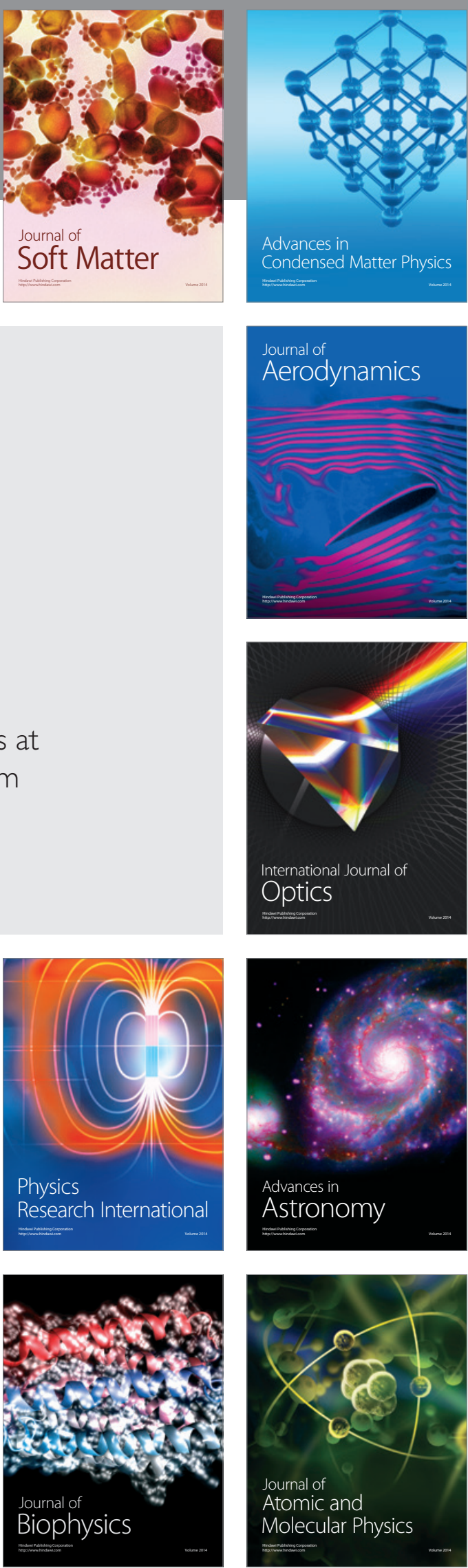